visual estimates, but that he took the proper precautions to eliminate the very effects which Professor Scheiner assumes he did not eliminate, as well as other physiological effects which Professor Scheiner has not mentioned.

Further evidence as to the observer's state of mind is afforded on page 494 of my same paper. Concerning the nebula $\mathrm{BD}$. $-12 \% 172$ I wrote: The relative intensities of the light in the three discs (corresponding to three nebular lines) were estimated at $10,3,7$. A wedge photometer of increasing darkness was moved over the eyepiece at right angles to the line joining the three discs; and the fact that the disc at $486 \mathrm{r}(H \beta)$ disappeared before the disc at 5007 did, proves that the latter is the brighter.
This nebula is near the Orion Nebula, and the photometer was used on the lines in both. It confirmed the results on the Orion Nebula previously obtained with the grating. The wedge was of neutral tint and its absorption curve would probably be no steeper at $H \beta$ than the absorption curve for the prism.

It has been suggested that the question of variations in the Orion Nebula spectrum be verified. by photography. Ordinary dry plates cannot be used to advantage in this problem, since their curve of sensitiveness is very steep between $H \beta$ and $\lambda=5007$. The image of $H \beta$ is stronger than that of $\lambda=5007$ on my plates, even in the Trapezium region. Special plates, for which the curve between $H \beta$ and $\lambda=5007$ is flatter, should be employed.

Lick Observatory, 1898 July 22.

W. W. Campbell.

\title{
Beobachtungen des Cometen 1898 V (Giacobini Juni 18)
}

am 18 z. Refractor der Kais. Universitätssternwarte zu Strassburg i. E. von Dr. H. Kobold.

\begin{tabular}{|c|c|c|c|c|c|c|c|c|c|c|c|}
\hline \multicolumn{2}{|c|}{1898} & M.Z.Str. & \multirow{2}{*}{$\frac{\Delta \alpha}{+2^{\mathrm{m}} \mathbf{2 4}^{\mathrm{s}} \cdot \mathbf{8}_{3}}$} & \multirow{2}{*}{$\frac{\Delta \delta}{-4^{\prime}+8.1}$} & \multirow{2}{*}{$\frac{\mathrm{Vgl}}{10.4}$} & \multirow{2}{*}{$\frac{\alpha \text { app. }}{\text { I } 4^{\mathrm{h}} 57^{\mathrm{m}} 34^{5} \cdot 7^{\mathrm{I}}}$} & \multirow{2}{*}{$\frac{|\log p . \Delta|}{9.5 \circ 7 \mid}$} & \multirow{2}{*}{$\frac{\delta \text { app. }}{-10^{\circ} 20^{\prime} 42^{\prime \prime 1}}$} & \multirow{2}{*}{$\frac{\log p \cdot \Delta}{0.849}$} & \multirow{2}{*}{$\begin{array}{l}\text { Red. ad 1. app. } \\
+3: 35-14: 6\end{array}$} & \multirow{2}{*}{$\frac{*}{1}$} \\
\hline & & $I_{1}{ }^{h} 0^{m} 19^{3}$ & & & & & & & & & \\
\hline & & I $\quad 0 \quad 19$ & $-3 \quad 9.70$ & $-03^{2.2}$ & 10.4 & $1457 \quad 34.79$ & 9.507 & $\begin{array}{lll}-10 & 20 & 37.9\end{array}$ & 0.849 & $+3.3^{8}-14.3$ & 2 \\
\hline & & $10 \quad 44 \quad 35$ & +144.77 & +254.4 & 20.8 & 145149 & 9.498 & -94922 & & $+3.3^{\circ}-14.7$ & 3 \\
\hline & 18 & 10 366 & +342.67 & +253.2 & 20.8 & 144128.08 & 9.510 & -85155.3 & 0.845 & $+3.19-14.8$ & \\
\hline
\end{tabular}

Mittlere Oerter der Vergleichsterne.

\begin{tabular}{|c|c|c|c|c|c|c|c|}
\hline * & $\alpha \quad 1898.0$ & $\delta 1898.0$ & Autorität & * & $\alpha \times 898.0$ & $\delta 1898.0$ & Autorität \\
\hline $\mathbf{I}$ & $14^{\mathrm{h}} 55^{\mathrm{m}} \quad 6^{\mathrm{s}} \cdot 53$ & - $10^{\circ} 16^{\prime} \quad 9^{\prime \prime} 4$ & $M_{1} 10815$ & 3 & $14^{\mathrm{h}} 50^{\mathrm{m}} \quad 1^{5} .29$ & $-9^{\circ} 5^{2^{\prime}} \quad 1.8$ & A.N. $1637:$ Berlin Nr. 388 \\
\hline 2 & I $5 \bigcirc 4$ I.II & - I0 $19 \quad 51.4$ & $M_{1} 10925$ & 4 & $1437 \quad 42.22$ & -85433.7 & Paris 18104 \\
\hline
\end{tabular}

Der Comet erschien am 16. Juli bei guter Luft als äusserst schwache Nebelmasse, in deren vorangehendem Theile sich ein Kern $15^{\mathrm{m}}$ befand. Die Beobachtung war aber trotzdem nur mit grösster Anstrengung möglich. An den beiden anderen Tagen waren die Verhältnisse noch ungtunstiger.

Strassburg i. E., 1898 Juli 20.

H. Kobold.

Beobachtungen des Cometen 1898...(Brooks Oct. 20).

\begin{tabular}{|c|c|c|c|c|c|c|c|c|c|c|}
\hline 1898 & M. Ortszeit & $\Delta \alpha$ & $\Delta \delta$ & Vgl. & $\alpha$ app. & $\log p . \Delta$ & $\delta$ app. & $\log p \cdot \Delta$ & Red. ad 1. app. & $*$ \\
\hline
\end{tabular}

Auf der Sternwarte in Göttingen von Prof. W. Schur.

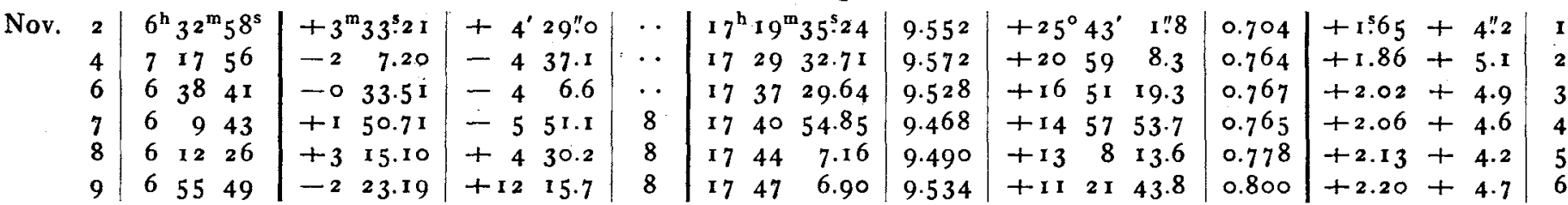

Nov. 2. Helle Masse mit concentrischer Verdichtung, Durchmesser 70". - Nov. 4. Hell, ohne Schweif. - Nov. 6. Comet erheblich schwăcher, Luft etwas dunstig. - Nov. 8. Comet schwächer, aber noch sehr gut zu beobachten.

Auf der Grossherzogl. Sternwarte in Jena von Prof. O. Knopf.

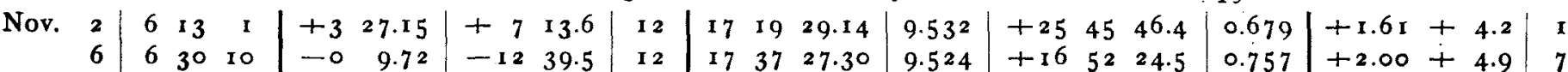

Die Beobachtungen sind mit dem Glaskreismikrometer (helle Kreislinien im dunklen Feld) angestellt. - Nov. 6. $\delta$ sicherer als $\alpha$. 